\title{
Recent hadron production measurements performed by the NA61/SHINE collaboration
}

\author{
Alexander E. Hervé for the NA61/SHINE Collaboration ${ }^{\mathrm{a}}$ \\ Institut für Kernphysik, Karlsruher Institut für Technologie, Postfach 3640, 76021 Karlsruhe, Germany
}

\begin{abstract}
NA61/SHINE is a fixed target experiment which uses secondary beams produced at the CERN SPS. Hadron-hadron interactions have been recorded at beam momenta between 13 and $350 \mathrm{GeV} / \mathrm{c}$ with a wide-acceptance spectrometer.

Recent measurements released by the NA61/SHINE experiment are of significant importance for a precise prediction of the J-PARC neutrino beam used for the T2K experiment and for interpretation of EAS data. These measurements also provide a large amount of input for tuning of hadron production models in MonteCarlo generators.

The latest measurements of pion-carbon and proton-carbon interactions will be reviewed.
\end{abstract}

\section{The NA61/SHINE experiment}

NA61/SHINE ${ }^{1}$ [1] is a multi-purpose fixed target experiment to study hadron production in hadron-nucleus and nucleus-nucleus collisions at the CERN Super Proton Synchrotron (SPS). Among its physics goals are precise hadron production measurements for improving calculations of the neutrino beam flux in the T2K and Fermilab neutrino oscillation experiment [2] as well as for more reliable simulations of hadronic interactions in air showers. Moreover, $\mathrm{p}+\mathrm{p}, \mathrm{p}+\mathrm{Pb}$ and nucleus+nucleus collisions are measured to study the properties of the onset of de-confinement and search for the critical point of strongly interacting matter (see e.g. Ref. [3]).

The layout of the NA61/SHINE detector is sketched in Fig. 1. The NA61/SHINE Collaboration uses large time-projection-chambers (TPCs) inherited from the NA49 experiment [4] to measure the charge and momentum of particles. The momentum resolution, $\sigma(1 / p)=\sigma(p) / p^{2}$, is about $10^{-4}(\mathrm{GeV} / \mathrm{c})^{-1}$ at full magnetic field and the tracking efficiency is better than $95 \%$. A set of scintillation and Cherenkov counters as well as beam position detectors upstream of the spectrometer provide timing reference, identification and position measurements of the incoming beam particles. Particle identification is achieved by measuring the energy loss along the tracks in the TPCs and by determining their velocity from the time of flight provided by large scintillator walls placed downstream of the TPCs. The centrality of nucleus-nucleus collisions can be estimated using the measurement of the energy of projectile spectators with a calorimeter [5] located behind the time of flight detectors. For nucleon-nucleus collisions, the centrality is determined by counting low momentum particles from the target (so called 'gray protons') with a small TPC around the target [6].

\footnotetext{
${ }^{a}$ http://shine.web.cern.ch/content/author-list

${ }^{1}$ SHINE = SPS Heavy Ion and Neutrino Experiment.
}

Data taking with the NA61/SHINE experiment started in 2007. After a first run with proton on carbon at $31 \mathrm{GeV} / \mathrm{c}$, the data acquisition system was upgraded during 2008 to increase the event recording rate by a factor of $\approx 10$ [7]. In recent years, a wealth of data has been recorded by the experiment at beam momenta ranging from 13 to $350 \mathrm{GeV} / \mathrm{c}$ with various beam particles and targets. In this paper we present results obtained from the data relevant for neutrino oscillations and the interpretation of air showers at ultra-high energies.

\section{Measurements of $p+C$ interactions for the improvement of neutrino flux calculations}

Data for the T2K experiment were collected in 2007, 2009 and 2010 by using a $31 \mathrm{GeV} / \mathrm{c}$ proton beam and 2 targets: a thin carbon target and a T2K replica target. Measurements with the thin target are used for constraining primary proton-carbon interactions which account for around $60 \%$ of the $\mathrm{T} 2 \mathrm{~K}$ neutrino flux. In contrast to the thin target, measurements with the $\mathrm{T} 2 \mathrm{~K}$ replica target include primary interactions and re-interactions in the target which account for more than $90 \%$ of the T2K neutrino flux. Results of the measurements performed in 2007 were published [8-11] and used for the $\mathrm{T} 2 \mathrm{~K}$ beam simulation. Results of the thin and T2K replica target measurements performed in 2009 have been recently published $[12,13]$ and will be discussed in the next two subsections. Analysis of the T2K replica target data taken in 2010 is ongoing.

\subsection{Hadron spectra in proton-carbon at $31 \mathrm{GeV} / \mathrm{c}$}

In the analysis of the data, different particle identification techniques were used in order to maximize coverage of the phase space and cross-check the results. For charged hadrons, we used 3 different analysis techniques: $\mathrm{dE} / \mathrm{dx}$ identification, combined $\mathrm{dE} / \mathrm{dx}$ and time-of-flight 


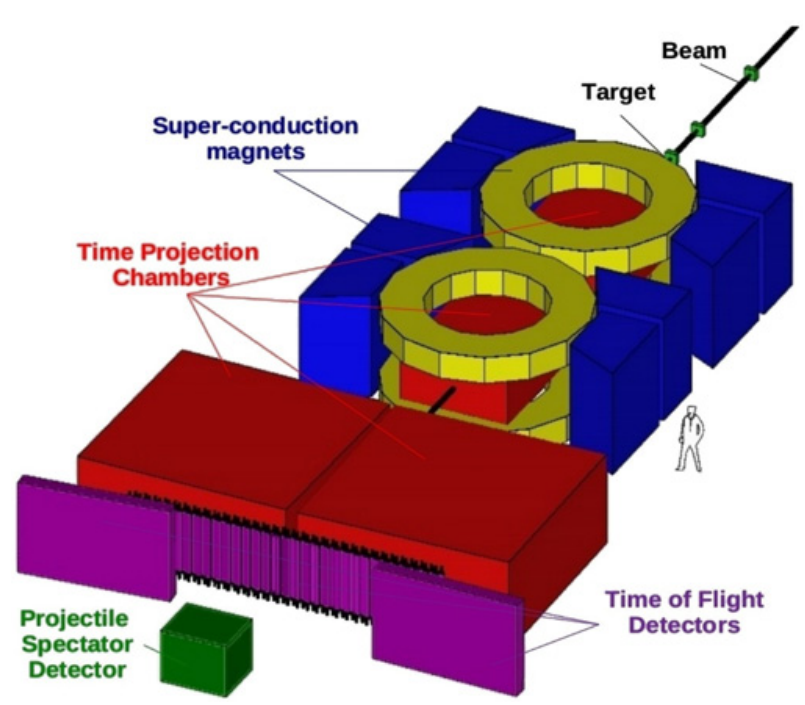

Figure 1. Schematic layout of the NA61/SHINE experiment.

identification and no identification (just for $\pi^{-}$). Examples of the first two methods can be seen in Fig. 2. This allows us to measure spectra in the regions of the phase space where one technique fails (for example in the region where Bethe-Bloch curves overlap). Spectra of the neutral hadrons have been extracted by fitting the invariant mass of the decay candidates in the detector. All results have been fully corrected with the Monte Carlo. Systematic uncertainty for the $\pi^{ \pm}$spectra is around $5 \%$, while for other hadrons it is up to $15 \%$. A detailed description of the different analysis techniques and overview of the systematic uncertainties can be found in [12]. The results are presented in the form of differential multiplicities as required for the $\mathrm{T} 2 \mathrm{~K}$ collaboration. Comparisons of the results with various MC model predictions (VENUS 4.12 [14,15], EPOS 1.99 [16], GiBUU 1.6 [17,18] and several GEANT4 physics lists) were also made. In Fig. 3 $\mathrm{K}^{ \pm}$spectra are presented and compared with the model predictions. In this case, EPOS 1.99 gives the most accurate description of the spectra. Spectra of $\pi^{ \pm}, \mathrm{K}^{ \pm}$, $\mathrm{p}, \mathrm{K}_{S}^{0}$ and $\Lambda$ with the $\mathrm{MC}$ predictions can be found in [12]. It is important to note that none of the models give accurate predictions for all particle spectra. Presented results are being used in the $\mathrm{T} 2 \mathrm{~K}$ beam simulation. The total uncertainty of the $\mathrm{T} 2 \mathrm{~K}$ neutrino flux after the reweighting of the flux with the thin target measurements is reduced down to $10 \%$. To reach the $\mathrm{T} 2 \mathrm{~K}$ goal of $5 \%$, accurate re-weighting which includes re-interactions in the $\mathrm{T} 2 \mathrm{~K}$ target is necessary. For this reason, hadron spectra exiting the surface of the T2K replica target are needed.

\subsection{Spectra of charged pions in the proton + $\mathrm{T} 2 \mathrm{~K}$ replica target interactions at $31 \mathrm{GeV} / \mathrm{c}$}

Measurements of the hadron spectra coming from the surface of the T2K replica target have one major difference with respect to the thin target measurements. Since neutrino flux depends on the position along the target surface from which neutrino parent particles are coming, the target is divided into 5 longitudinal bins and the downstream target face. TPC tracks were extrapolated towards the target surface and stored in the longitudinal bins. For each longitudinal bin, analysis similar to the analysis of the thin target dataset was performed. The results are shown in the form of differential multiplicities in the bins of momentum, polar angle and longitudinal position along the target surface. Spectra of the $\pi^{+}$exiting the target surface together with FLUKA 2011 prediction are shown in Fig. 4. Each column represents a different longitudinal bin. The largest systematic contribution is due to the track extrapolation and can be up to $10 \%$. Full results for the $\pi^{ \pm}$and detailed description of the analysis can be found in [13].

The ratio of $v_{\mu}$ flux re-weighted with the thin target procedure over the T2K replica target flux at SK is shown in Fig. 5. At low neutrino energy the thin target procedure predicts a $10 \%$ larger value of the flux which is however consistent with the replica target prediction within one standard deviation. At high neutrino energy, the ratio tends to one. This is due to the fact that within this energy range the dominant part of the $v_{\mu}$ spectra comes from kaons and thus is not affected by the re-weighting procedure on pion spectra. Extraction of the $\mathrm{K}^{ \pm}$spectra from this dataset is not possible because of the low statistics, but analysis of the high-statistics $\mathrm{T} 2 \mathrm{~K}$ replica target dataset recorded in 2010 is ongoing.

\section{Measurements of $\pi+C$ interactions for the interpretation of cosmic ray air showers}

During the 2009 run, NA61/SHINE also recorded data with negatively charged pion beams at 158 and $350 \mathrm{GeV} / \mathrm{c}$ on a thin carbon target. These measurements will provide particle-production data for the tuning of hadronic interaction models used in air-shower simulations. The first preliminary results have already been released [19-22] and are summarized below.

\subsection{Spectra of $\rho^{0}$ mesons}

The measurement of resonances in $\pi+\mathrm{C}$ is useful to constrain the production of $\rho^{0}$ meson, which is important to predict the number of muons observed in air showers due to the leading particle effect (see e.g. Ref. [23]).

In the inclusive $\pi^{+} \pi^{-}$invariant mass spectra there is a large combinatorial background, which dominates over the effective mass distributions of individual resonances. The method used to estimate the background is the so called charge mixing, which uses the $\left(\pi^{+} \pi^{+}+\pi^{-} \pi^{-}\right)$ mass spectra as an estimate of the background.

The fitting procedure uses templates of the $\pi^{+} \pi^{-}$ mass distribution for each resonance. These templates are constructed by passing simulated $\pi+\mathrm{C}$ interactions, generated with the Epos1.99 [16] hadronic interaction model using CRMC [24] (v1.5.3), through the full NA61 detector Monte Carlo chain. All the cuts that are applied to the data are also applied to the templates. This method of using templates allows fitting, in addition to resonances with 2-body decays, resonances with dominant three body decays, such as the $\omega$, and resonances with non- $\pi^{+} \pi^{-}$ decays, such as the $\mathrm{K}^{* 0}$.

An example of one of these fits can be seen in Fig. 6 . The templates in the fit are the background found from 

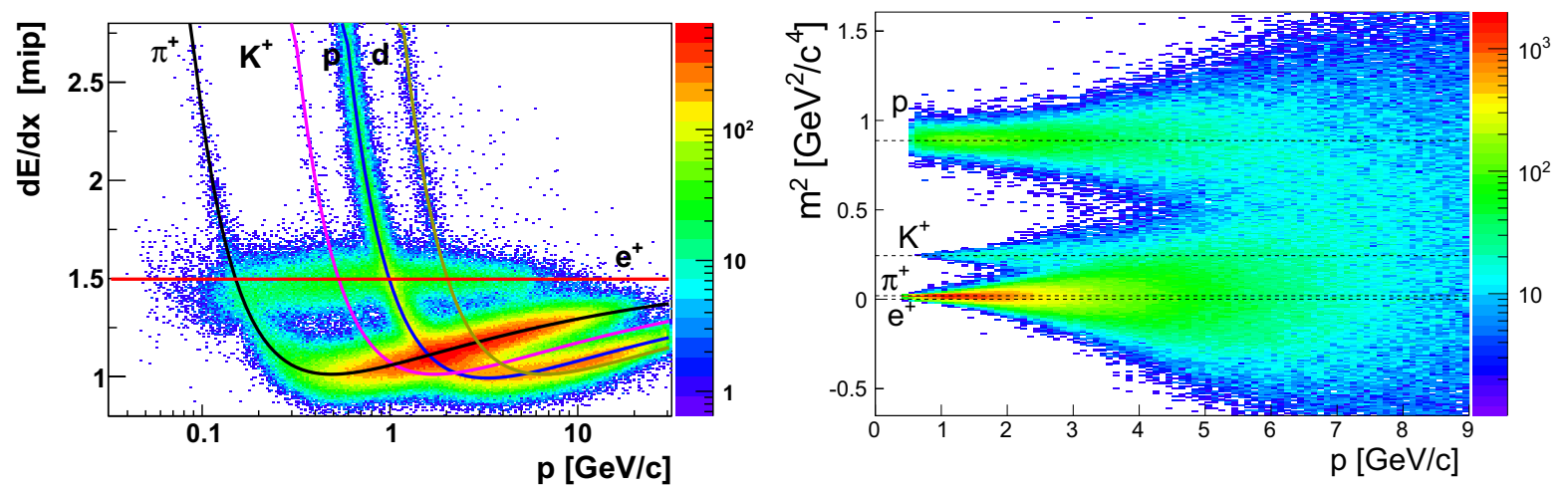

Figure 2. Examples of PID capabilities of the NA61/SHINE spectrometer for positively charged particles. Left: specific energy loss in the TPCs as a function of momentum. Curves show parameterizations of the mean $d E / d x$ calculated for different particle species. Right: mass squared, derived from the ToF-F measurement and the fitted path length and momentum, versus momentum. The lines show the expected mass squared values for different particles.
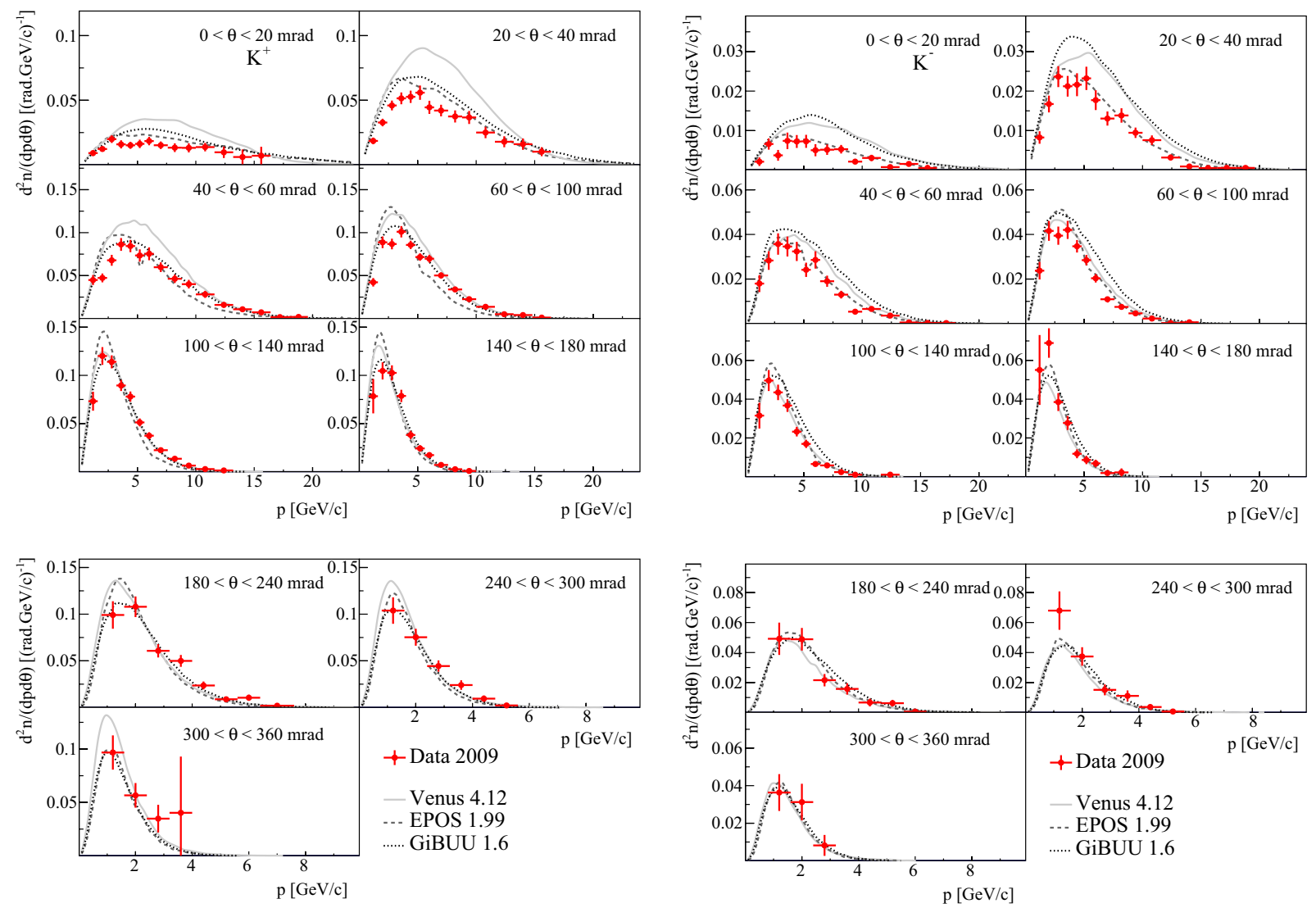

Figure 3. Comparison of measured $\mathrm{K}^{+}$(left) and $\mathrm{K}^{-}$(right) spectra with model (VENUS, EPOS, GIBUU) predictions. Distributions are normalized to the mean $\mathrm{K}^{+}\left(\mathrm{K}^{-}\right)$multiplicity in all production $\mathrm{p}+\mathrm{C}$ interactions. The vertical error bars on the data points show the total (stat. and syst.) uncertainty. The horizontal bars indicate the bin size in momentum.

charge mixing and the following resonances: $\rho^{0}, \mathrm{~K}^{* 0}, \omega$, $\mathrm{f}_{2}, \mathrm{f}_{0}(980), \mathrm{a}_{2}, \eta$ and $\mathrm{K}_{S}^{0}$.

The average multiplicity of $\rho^{0}$ mesons is presented in Fig. 7. Also shown are predictions by Epos1.99 [16], DPMJet3.06 [25], Sibyll2.1 [26], QGSJetII-04 [27] and EposLHC [28]. It can be seen that there is an underestimation of the $\rho^{0}$ yield for almost all hadronic interaction models, with the exception of QGSJetII-04 for $x_{\mathrm{F}}>0.8$. It is interesting to note that while QGSJetII-04 and EposLHC were tuned to NA22 $\pi^{+}+\mathrm{p}$ data [29], there is an underestimation in $\pi^{-}+\mathrm{C}$.
The systematic error is dominated by the background estimates, up to $14 \%$, whereas the other errors are less than $4 \%$. Other sources of uncertainty, such as using templates from a different model, are found to be much smaller.

\subsection{Spectra of charged pions}

The measured average multiplicities of charged pions are shown in Fig. 8 and the measurements are compared to predictions of pion production in $\pi^{-}+\mathrm{C}$ at $158 \mathrm{GeV} / \mathrm{c}$ from hadronic interaction models in Fig. 9. As can be seen, none of the generators describe the data well. 


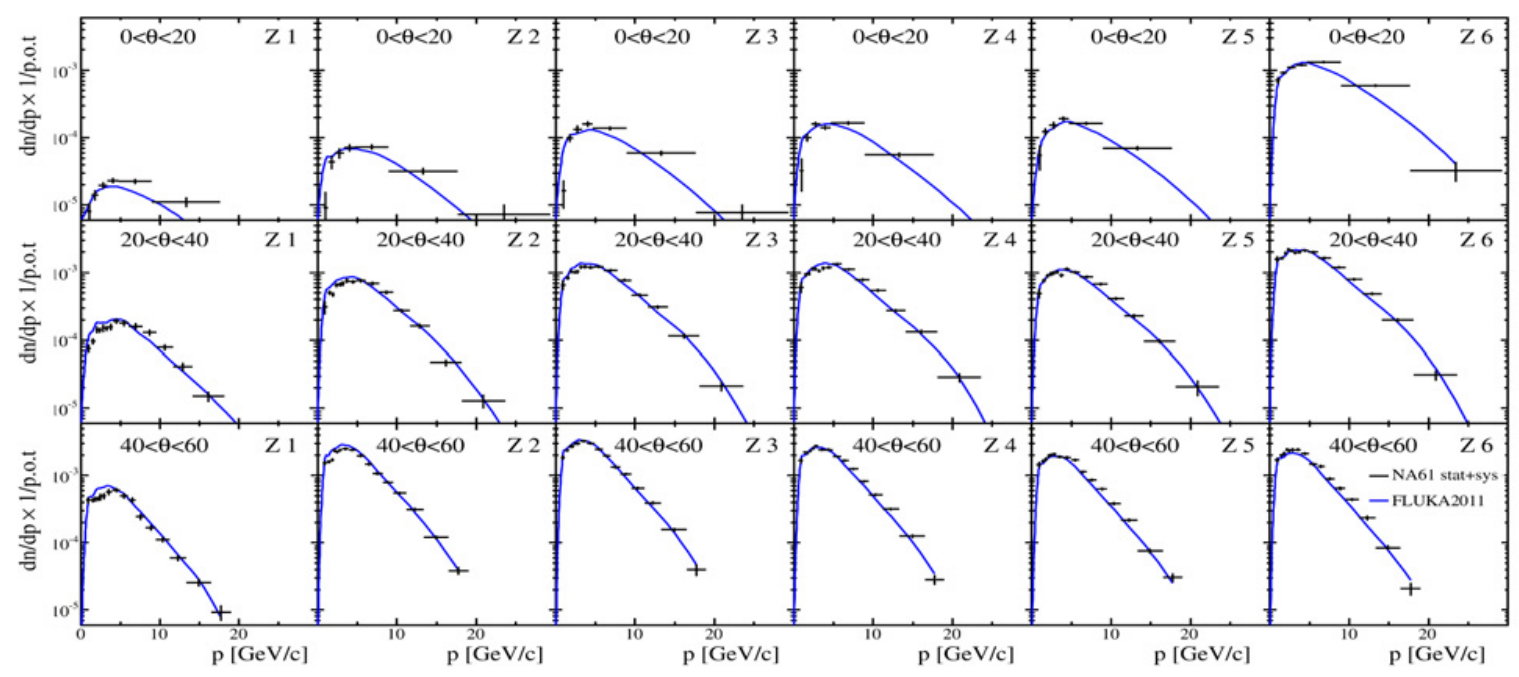

Figure 4. Spectra of positively charged pions at the surface of the T2K replica target, in the polar angle range from 0 to $60 \mathrm{mrad}$, and for the six longitudinal bins as a function of momentum. The normalization is per proton on target. The prediction from FLUKA2011 is overlaid.

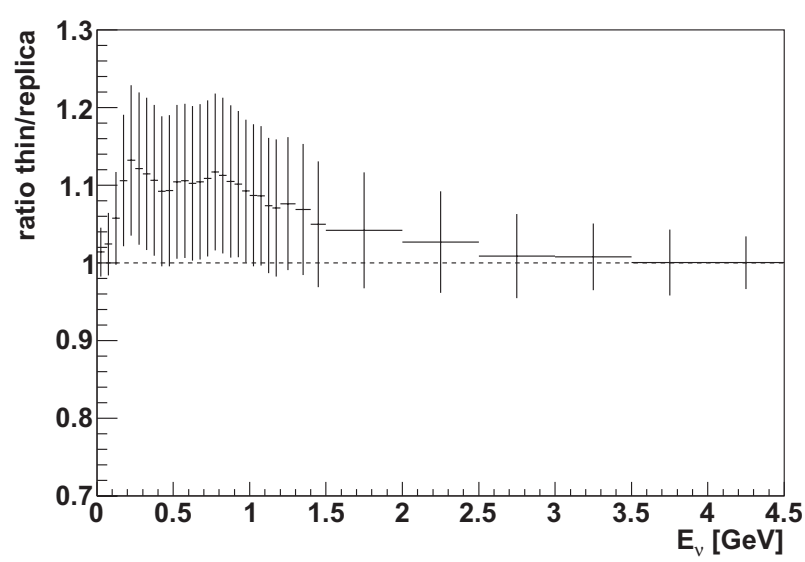

Figure 5. Ratio of the $v_{\mu}$ flux at SK re-weighted with the thin target procedure to the $\mathrm{p}+(\mathrm{T} 2 \mathrm{~K} \mathrm{RT})$ at $31 \mathrm{GeV} / \mathrm{c}$ flux. For the latter, pion spectra presented in [13] have been used in the reweighting. The dominant part of the $v_{\mu}$ spectra at high $E_{v}$ comes from kaons and thus is not affected by the re-weighting of the pion spectra.

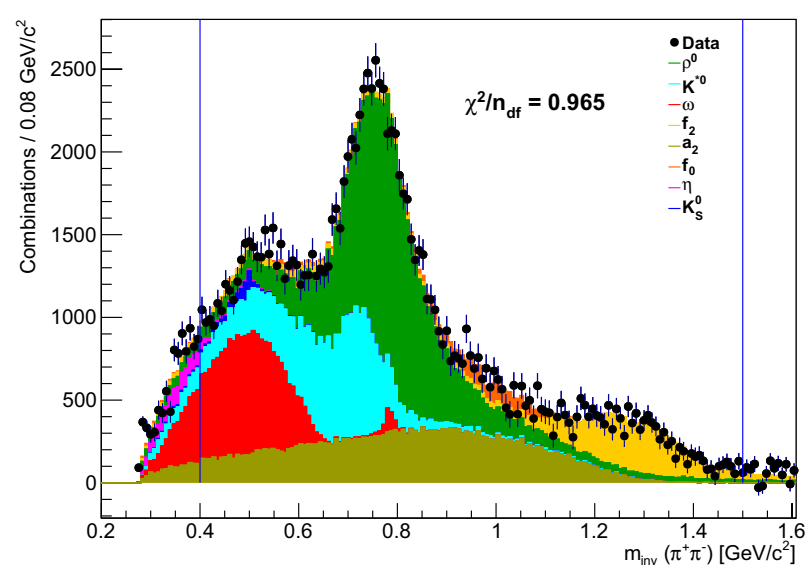

Figure 6. $\pi^{+} \pi^{-}$invariant mass distribution in $\pi^{-}+\mathrm{C}$ interactions at $158 \mathrm{GeV} / \mathrm{c}$ in the range $0.4<x_{\mathrm{F}}<0.5$. Dots with error bars denote the data and the fitted resonance templates are shown as filled histograms. The vertical lines indicate the range of the fit.

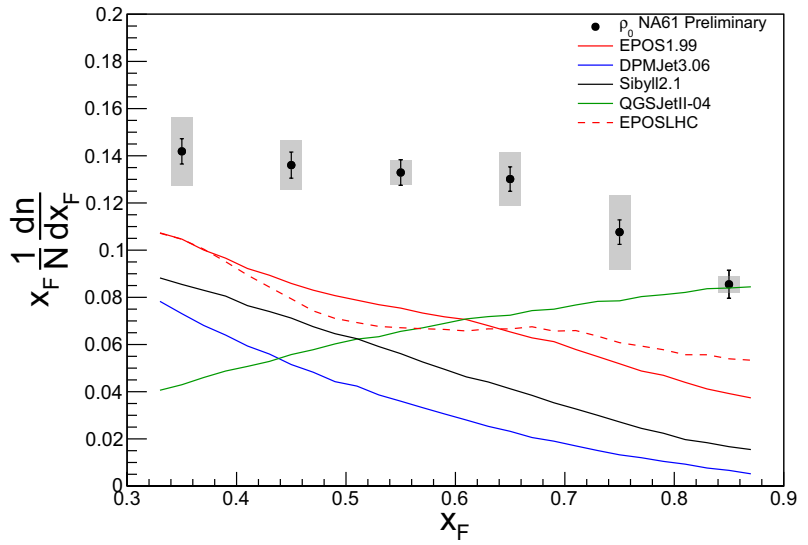

Figure 7. Average multiplicity of the $\rho^{0}$ meson in $\pi+C$ at $p_{\text {beam }}=158 \mathrm{GeV} / \mathrm{c}$ as a function of Feynman- $x$. The bars show the statistical errors; the bands indicate systematic errors. The lines depict predictions of hadronic interaction models: red - Epos1.99, blue - DPMJet3.06, black - Sibyl12.1, green QGSJetII-04, dashed red - EposLHC.

These spectra were obtained within a fiducial phase space of the NA61/SHINE detector, for which the detection and selection efficiency for charged tracks is close to unity, and then corrected for the detector acceptance, selection efficiency, feed-down from weak decays and re-interactions in the target. The latter two corrections are currently estimated using model predictions (Epos1.99, QGSJetII-04, DPMJet3.06) and they are typically well below $5 \%$, but can reach up to $20 \%$ at low particle momenta. Overall, the systematic uncertainty of the corrected number of tracks, $\Delta n^{\prime}$, is estimated to be $\leq 7 \%$.

The average multiplicity of particles produced within a $p / p T$-bin is then obtained by dividing $\Delta n^{\prime}$ by the total number of events in which an interaction occurred, $N_{\text {prod. }}$. $N_{\text {prod }}$ is estimated by extrapolating the number of recorded interaction triggers to full phase space. The correction amounts to $(92.5 \pm 3.5) \%$ at $158 \mathrm{GeV} / \mathrm{c}$ and $(92.5 \pm 4.0) \%$ at $350 \mathrm{GeV} / \mathrm{c}$, where the uncertainty was 
NA61/SHINE preliminary
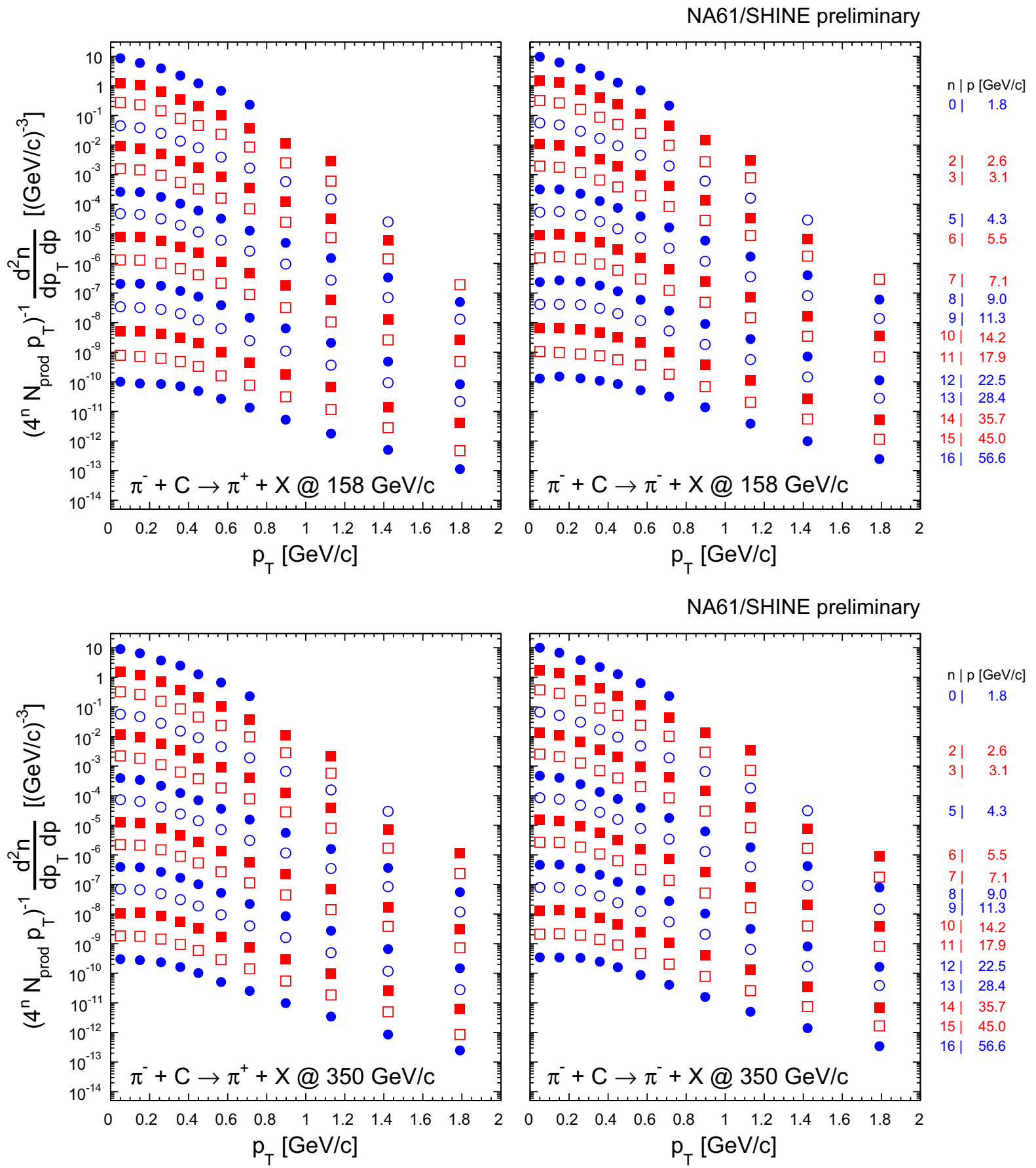

NA61/SHINE preliminary

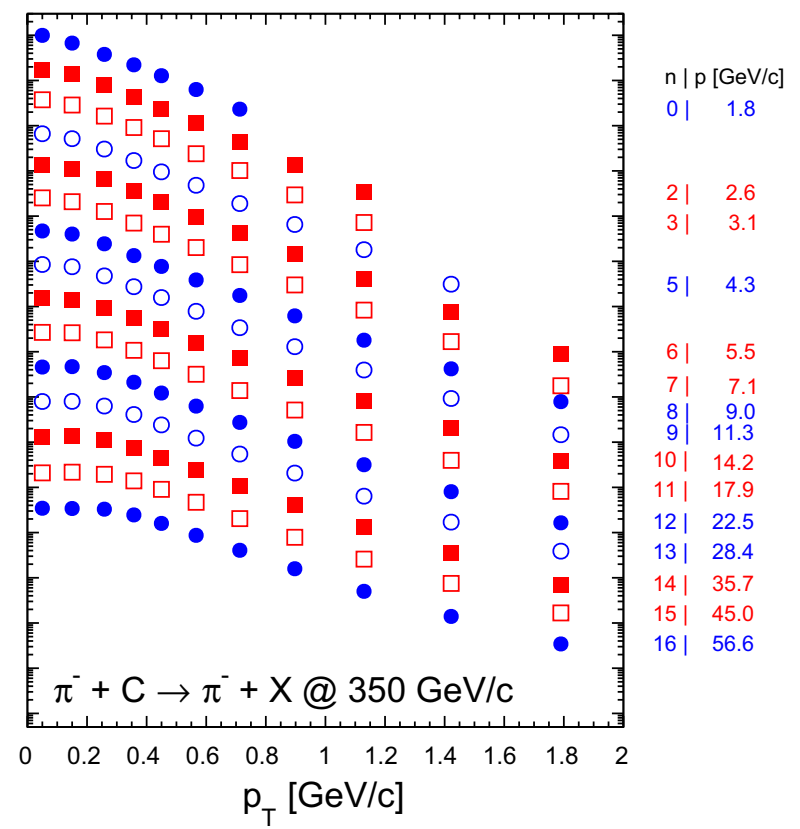

Figure 8. Inclusive spectra of charged pions in $\pi^{-}+\mathrm{C}$ interactions at beam energies of 158 and $350 \mathrm{GeV} / c$. For better visibility, the spectra from the $n^{\text {th }}$ momentum bin are multiplied by a factor of $1 / 4^{n}$. The momentum increases from top to bottom as indicated in the legend on the right. Systematic errors are less than $7 \%$

derived by running different generators to evaluate the correction.

\section{Conclusions and outlook}

In this article, results from pion-carbon and proton-carbon interactions, measured with the multi-purpose experiment NA61/SHINE at the CERN SPS, have been summarised. These results are of importance for the modelling of cosmic ray air showers and neutrino flux predictions.

Measurements of charged pion, kaon, proton, $\mathrm{K}_{S}^{0}$ and $\Lambda$ production in $\mathrm{p}+\mathrm{C}$ interactions at $31 \mathrm{GeV} / \mathrm{c}$ released by NA61/SHINE are of significant importance and were already employed for precise predictions of the J-PARC neutrino beam used for the first stage of the T2K experiment. The recently published results of the 2009 measurements presented here will further improve the precision of these predictions.

Preliminary results from the $\pi$-carbon interactions suggest the high-energy interaction models need further tuning to reproduce the charged pion and $\rho^{0}$ spectra.

Future measurements for neutrino physics will include precise measurements of hadron emission from a replica target of the Long-Baseline Neutrino Facility (LBNF) at Fermilab required for the Deep Underground Neutrino Experiment (DUNE). The NA61/SHINE measurements 

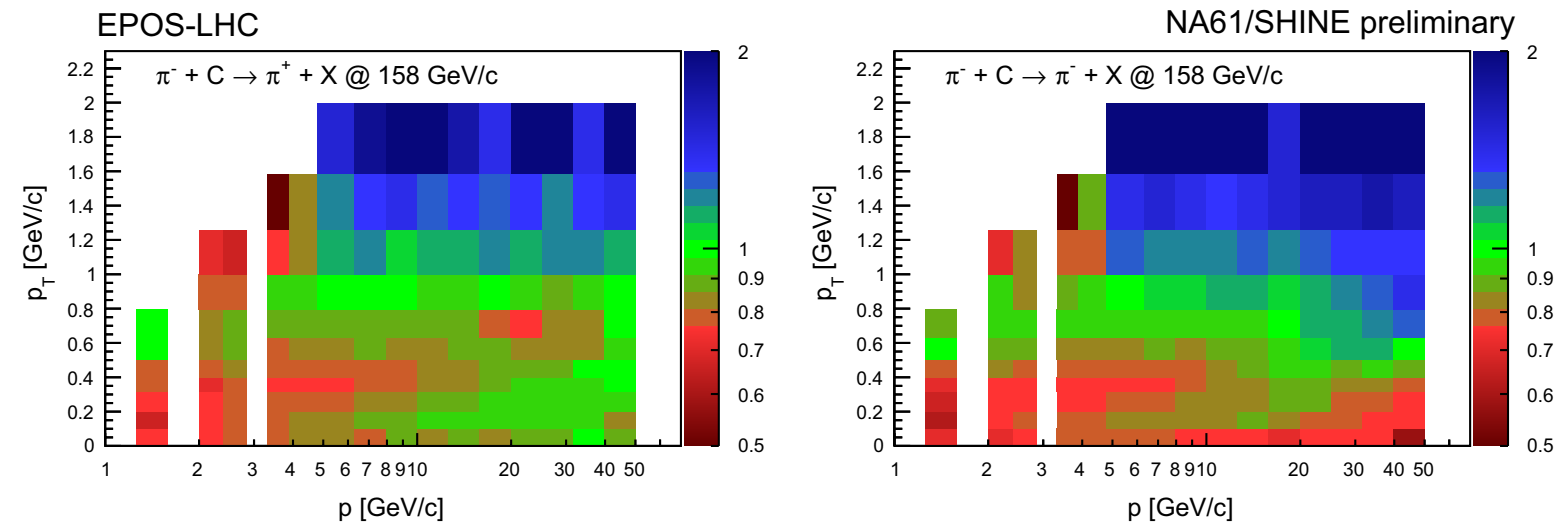

Sibyll-2.1
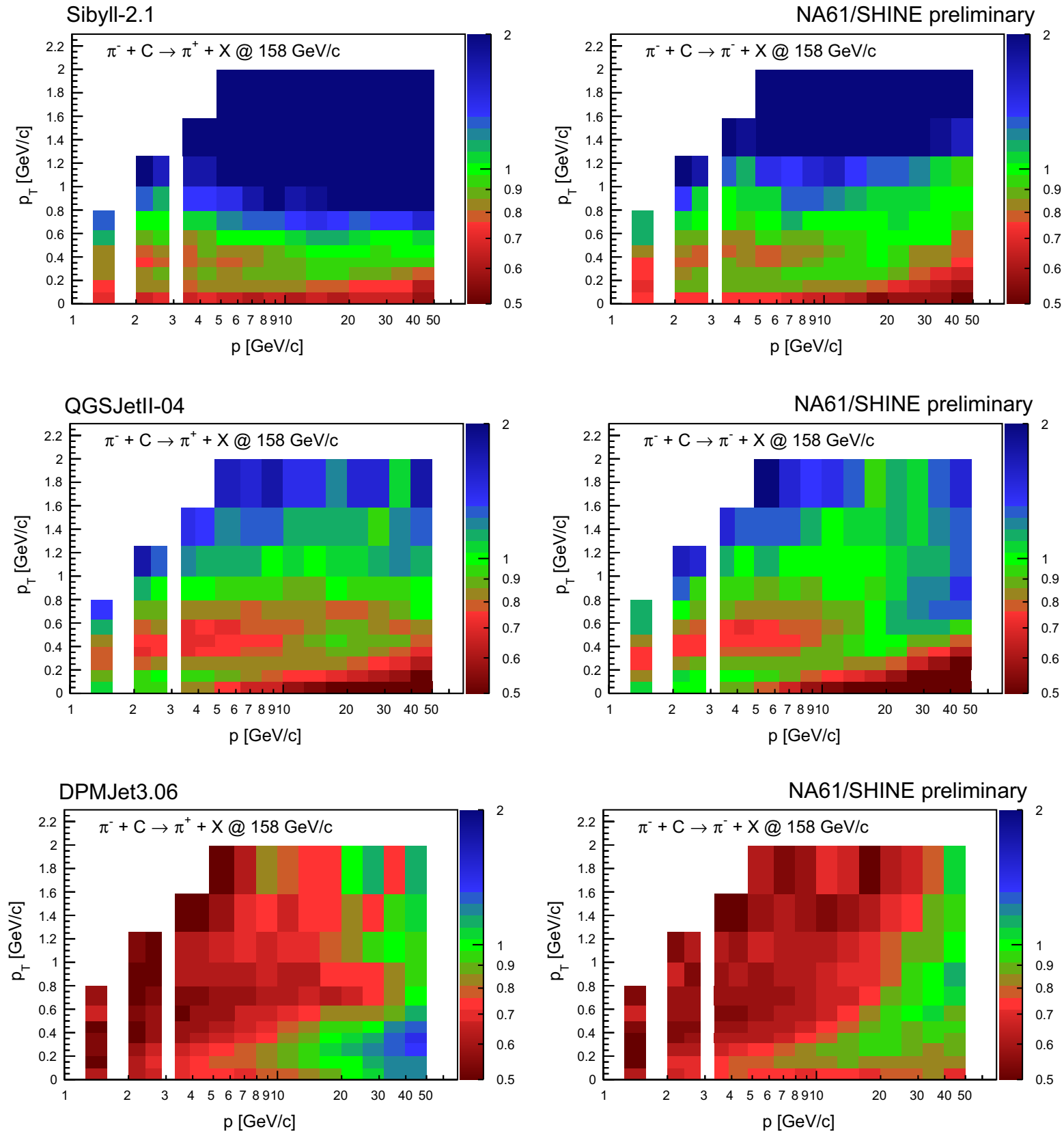

Figure 9. Comparison of the measured production spectra of charged pions to predictions from hadronic interaction models used for the interpretation of cosmic ray data [25-28]. The colors denote the ratio of data to simulation and the color scale is truncated at 0.5 and 2 . The two empty $p$-bins at $p \lesssim 2 \mathrm{GeV} / \mathrm{c}$ and $p \gtrsim 3 \mathrm{GeV} / \mathrm{c}$ are momenta excluded from the analysis due to the ambiguity in identification of pions. 
with thin and replica targets for $\mathrm{T} 2 \mathrm{~K}$ demonstrated a need for precise data on hadron productions from the surface of targets of future long baseline neutrino oscillation experiments, e.g. DUNE and Hyper-K.

The rich data set of NA61/SHINE will also be very useful to reduce uncertainties in air shower calculations and increase the knowledge on interactions in the late stages of the shower development below a TeV.

This work was supported by the Hungarian Scientific Research Fund (grants OTKA 68506 and 71989), the János Bolyai Research Scholarship of the Hungarian Academy of Sciences, the Polish Ministry of Science and Higher Education (grants 667/NCERN/2010/0, NN 202484339 and NN 20223 1837), the Polish National Center for Science (grants 2011/03/N/ST2/03691, 2012/04/M/ST2/00816, 2013/11/N/ST2/03879, 2014/13/N/ST2/ 02565), the Foundation for Polish Science - MPD program, cofinanced by the European Union within the European Regional Development Fund, the Federal Agency of Education of the Ministry of Education and Science of the Russian Federation (SPbSU research grant 11.38.242.2015), the Russian Academy of Science and the Russian Foundation for Basic Research (grants 08-02-00018, 09-02-00664 and 12-02-91503-CERN), the Ministry of Education, Culture, Sports, Science and Technology, Japan, Grant-in-Aid for Scientific Research (grants 18071005, 19034011, 19740162, 20740160 and 20039012), the German Research Foundation (grant GA 1480/2-2), the EU-funded Marie Curie Outgoing Fellowship, Grant PIOF-GA-2013-624803, the Bulgarian Nuclear Regulatory Agency and the Joint Institute for Nuclear Research, Dubna (bilateral contract No. 4418-115/17), Ministry of Education and Science of the Republic of Serbia (grant OI171002), Swiss Nationalfonds Foundation (grant 200020117913/1) and ETH Research Grant TH-01 07-3.

\section{References}

[1] N. Abgrall et al. [NA61/SHINE Collaboration], JINST 9 (2014) P06005

[2] K. Abe et al. [T2K Collaboration], Nucl. Instrum. Meth. A 659 (2011) 106

[3] M. Gazdzicki, M. Gorenstein, P. Seyboth, Acta Phys. Polon. B42 (2011) 307

[4] S. Afanasev et al. [NA49 Collaboration], Nucl. Instrum. Meth. A 430 (1999) 210
[5] M.B. Golubeva, F.F. Guber, A.P. Ivashkin, A.B. Kurepin, V.N. Marin, A.S. Sadovsky and O.A. Petukhov, Phys. Atom. Nucl. 75 (2012) 673

[6] K. Marton, G. Kiss, A. László and D. Varga, Nucl. Instrum. Meth. A 763 (2014) 372

[7] A.László et al., Nucl. Instrum. Meth. A 798 (2015) 1

[8] N. Abgrall et al. [NA61/SHINE Collaboration], Phys. Rev. C 84 (2011) 034604

[9] N. Abgrall et al. [NA61/SHINE Collaboration], Phys. Rev. C 85 (2012) 035210

[10] N. Abgrall et al. [NA61/SHINE Collaboration], Phys. Rev. C 89 (2014) 025205

[11] N. Abgrall et al. [NA61/SHINE Collaboration], Nucl. Instrum. Meth. A 701 (2013) 99

[12] N. Abgrall et al. [NA61/SHINE Collaboration], Phys. Rev. C 76 (2016) 84

[13] N. Abgrall et al. [[NA61/SHINE Collaboration], Eur. Phys. J. C 76 (2016) 617

[14] K. Werner, Nucl. Phys. A 525 (1991) 501

[15] K. Werner, Phys. Rept. 232 (1993) 87

[16] T. Pierog and K. Werner, Phys. Rev. Lett. 101 (2008) 171101

[17] O. Buss et al., Phys. Rept. 512 (2012) 1

[18] K. Gallmeister and U. Mosel, Nucl. Phys. A 826 (2009) 151

[19] M. Unger [NA61/SHINE Collaboration], EPJ Web Conf. 52 (2013) 01009

[20] L. Zambelli (2013) Ph.D. Thesis, University of Paris VII

[21] B. A. Popov, EPJ Web Conf. 99 (2015) 02002

[22] A. Herve [NA61/SHINE Collaboration], PoS ICRC 2015 (2016) 330 [arXiv: 1509. 06586 [nucl-ex]]

[23] H.-J. Drescher, Phys. Rev. D 77 (2008) 056003

[24] https://web.ikp.kit.edu/rulrich/crmc.html

[25] S. Roesler, R. Engel and J. Ranft, [arXiv:hep$\mathrm{ph} / 0012252]$

[26] E.J. Ahn et al., Phys. Rev. D 80 (2009) 094003

[27] S. Ostapchenko, Phys. Rev. D 83 (2011) 014018

[28] T. Pierog, I. Karpenko, J. M. Katzy, E. Yatsenko and K. Werner, Phys. Rev. C 92, no. 3 (2015) 034906

[29] N.M. Agababyan et al. [EHS/NA22 Collaboration], Z. Phys. C 46 (1990) 387 